\title{
A Monte Carlo study on the radio-sensitization effect of gold nanoparticles in brachytherapy of prostate by ${ }^{103} \mathrm{Pd}$ seeds
}

\author{
Amir Ghasemi JANGJOO ${ }^{1}$, Hosein GHIASI ${ }^{2, a}$, Asghar MESBAHI ${ }^{2,3}$ \\ ${ }^{I}$ Radiation Oncology Department, Imam Reza Hospital, Tabriz, Iran \\ ${ }^{2}$ Medical Radiation Sciences Research Team, Tabriz University of Medical Sciences, Tabriz, Iran \\ ${ }^{3}$ Medical Physics Department, Medical School, Tabriz University of Medical Sciences, Tabriz, Iran \\ ${ }^{a}$ E-mail address: hoseinghiasi62@gmail.com
}

(received 29 September 2018; revised 5 February 2019; accepted 7 March 2019)

\begin{abstract}
${ }^{103} \mathrm{Pd}$ seed is being used for prostate brachytherapy. Additionally, the dose enhancement effect of gold nanoparticles (GNP) has been reported in previous studies. The aim of this study was to characterize the dosimetric effect of gold nanoparticles in brachytherapy with a ${ }^{103} \mathrm{Pd}$ source. Two brachytherapy seeds including $103 \mathrm{Pd}$ source was simulated using MCNPX Monte Carlo code. The seeds' models were validated by comparing the MC with reported results. Then, GNPs $(10 \mathrm{~nm}$ in diameter) with a concentration of $7 \mathrm{mg} \mathrm{Au} / \mathrm{g}$ were simulated uniformly inside the prostate of a humanoid computational phantom. Additionally, the dose enhancement factor (DEF) of nanoparticles was calculated for both modeled brachytherapy seeds. A good agreement was found between the MC calculated and the reported dosimetric parameters. For both seeds, an average DEF of $23 \%$ was obtained in tumor volume for prostate brachytherapy. The application of GNPs in conjunction with ${ }^{103} \mathrm{Pd}$ seed in brachytherapy can enhance the delivered dose to the tumor and consequently leads to better treatment outcome.
\end{abstract}

Key words: ${ }^{103} \mathrm{Pd}$; prostate cancer; brachytherapy; Monte Carlo; gold nanoparticle.

\section{Introduction}

Brachytherapy plays a crucial role in the treatment of prostate cancer. Different radioactive sources are employed to deliver the prescribed dose properly to the prostate and lower the received dose to normal surrounding tissues. However, the studies on the optimization of the treatment method and enhancing the treatment outcome are still conducted. For example, Yang and Wang treated twenty patients with prostate cancer and used different sources including ${ }^{131} \mathrm{Cs},{ }^{103} \mathrm{Pd}$, and ${ }^{125}$ I seeds. Finally, they preferred ${ }^{131} \mathrm{Cs}$ because less seed needed [1]. Ververs $\mathbf{J}$ et al. conducted a comparative study in prostate brachytherapy with ${ }^{103} \mathrm{Pd}$. They concluded that based on clinical scenarios, planning with the CivaString source significantly reduced the number of required needles while delivering similar dose distributions to the prostate, urethra, and rectum. Planning was dramatically simplified, and optimization was replaced by simple guidelines that allowed the creation of high-quality treatment plans within minutes [2]. Rivard et al. studied ${ }^{103} \mathrm{Pd}$ seeds usage to the prostate gland cancer and calculated in detail the dosimetric parameters of a model of ${ }^{103} \mathrm{Pd}$ seeds for prostate brachytherapy. They studied the new designed CivaString and CivaThin sources in comparison to the encapsulated ${ }^{103} \mathrm{Pd}$ sources. In their designed seeds, dose distributions of both source types had minimal anisotropy in comparison to conventional ${ }^{103} \mathrm{Pd}$ seeds [3].

${ }^{103} \mathrm{Pd}$ seeds are the highly used radioactive sources applied in brachytherapy of prostate cancer. These sources emit low energy photons which make them preferred candidates for brachytherapy of prostate cancer. These sources provide a high dose region inside the tumor volume, while they deliver a very small amount of radiation dose to the critical peripheral organs such as bladder and rectum.

There are enormous studies on the dose enhancement effect of gold nanoparticles (GNP) in radiation therapy with radioactive sources [4-7]. It indicates the strong interest of researchers worldwide to use the brachytherapy sources with nanoparticles inside a tumor to improve the treatment outcome [8-12]. In fact, radiation dose enhancement of GNPs stems from higher probability of interaction of radiation with the gold atoms. In other words, the probability of photoelectric interaction which is dependent on the atomic number of a material increases significantly for gold atoms. Thus, production of the photoelectrons which deposit their energy in short distances relative to gold atoms causes a higher dose delivery to the medium containing a sufficient amount of GNPs [13-19]. Also, in microscopic studies, it was shown a high dose gradient region with a few $\mu \mathrm{m}$ distance around the GNPs which 
results in enhanced absorbed dose in the target organ [18-20]. GNPs and other nanoparticles have been studied for their potential dose enhancement effect in brachytherapy [21-23]. In Monte Carlo (MC) studies, a newly fabricated ${ }^{103} \mathrm{Pd}$ source was investigated for its dosimetric property for application in brachytherapy of prostate cancer [24-28]. They confirmed its dosimetric suitability for brachytherapy of prostate cancer. However, the dose enhancement factor of GNPs with this new source of ${ }^{103} \mathrm{Pd}$ and the other clinically used seed of ${ }^{125} \mathrm{I}$ have not been studied. It is worth to estimate their dose enhancement effect in the treatment of GNP-loaded prostate tumor.

The aim of this study was to characterize the dosimetric properties of two newly produced radioactive seeds including the IR01- ${ }^{103} \mathrm{Pd}$ source for application in prostate brachytherapy. Also, the dose enhancement of these seeds in conjunction with GNPs was evaluated for prostate brachytherapy.

\section{Materials and Methods}

In the current study, the MCNPX MC (ver.2.7.0) code (Los Alamos National Laboratory) was employed for $\mathrm{MC}$ simulations. In Figure 1, the simulated IR01- ${ }^{103} \mathrm{Pd}$ seed was schematically shown. Four resin beads with a diameter of 0.06 $\mathrm{cm}$ containing the radioactive ${ }^{103} \mathrm{Pd}$ were used to construct the seed with a length of $0.45 \mathrm{~cm}$ length. Moreover, a cylindrical copper cell with $0.15 \mathrm{~cm}$ length was located at the center of the seed. The composition of resin beads (density of $1.14 \mathrm{~g} / \mathrm{cm}^{3}$ ) was made of Hydrogen (8\%), Carbon (90\%), Nitrogen $(0.3 \%)$, Chlorine $(0.7 \%)$ and $\mathrm{Pd}(1 \%)$. The radiation spectrum of ${ }^{103} \mathrm{Pd}$ and cross-section of TG43U1 publication were used for source definition in the MC model [29]. The ${ }^{103} \mathrm{Pd}$ seed had an average energy of $21 \mathrm{keV}$ for photons, the half-life of 16.97 days. According to the updated AAPM Task group report No. 43, dosimetric characteristics of the both simulated seed as brachytherapy sources such as dose rate constant $\Lambda$ (cGy h ${ }^{-1}$ $\mathrm{U}^{-1}$ ), geometry function, radial dose function and anisotropy function calculated were estimated using MC method [30]. For dose calculations around sources, a water phantom with a dimension of $10 \times 10 \times 10 \mathrm{~cm}^{3}$ and a seed in its center were modeled (Figure 2). In order to score dose deposition around the seed, the lattice card was used and the water phantom was divided into some voxels with a dimension of $2 \times 2 \times 2 \mathrm{~mm}^{3}$. The dose deposition was scored using tally $* \mathrm{~F} 8$ which calculates the energy in terms of $\mathrm{MeV}$ per initial photon in each scoring cell. All dosimetric data were extracted from dose distribution data around the seed.

For dose enhancement calculations, a seed was simulated in the center of the prostate gland of Korean man computational phantom. Additionally, a concentration of $7 \mathrm{mg}$ Au/g GNPs (diameter $=10 \mathrm{~nm}$ ) was uniformly simulated in the prostate gland tissue using lattice and universe card properties in MCNPX code. All the MC calculated doses were relative and for absolute dose calculations, a prescribed dose of 145 Gy was

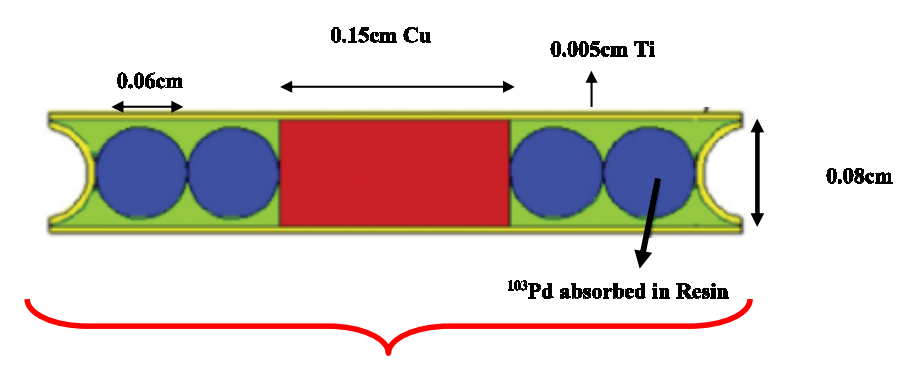

$0.45 \mathrm{~cm}$

Figure 1. MC simulated IR01- ${ }^{103}$ Pd brachytherapy seed.

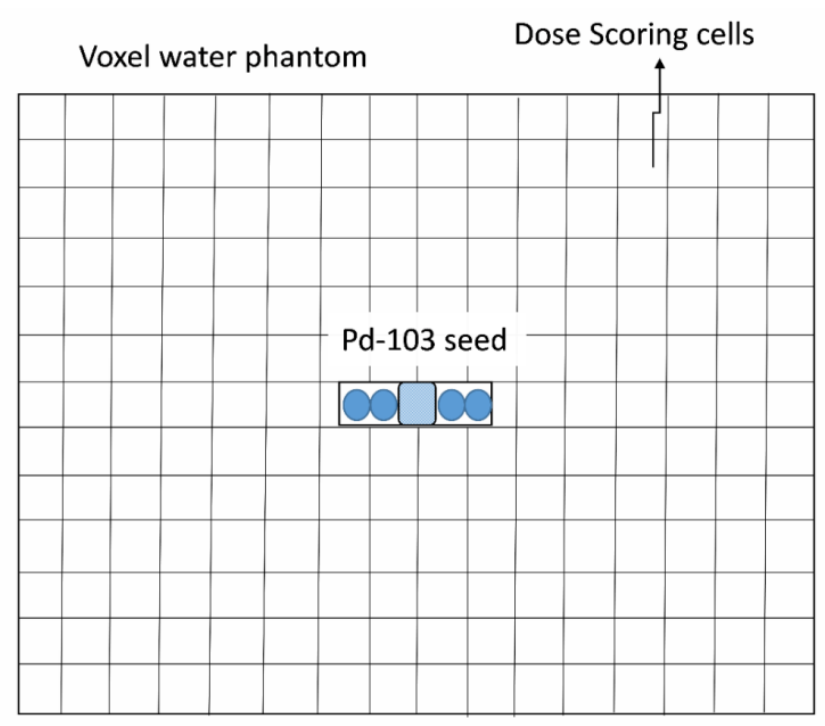

Figure 2. The schematic representation of geometry used for dose scoring around the ${ }^{103} \mathrm{Pd}$ seed. The lattice card of MCNPX code was used to produce $2 \times 2 \times 2 \mathrm{~mm}^{3}$ voxels around the seed.

considered for the cancerous prostate. The initial dose rate of a $19.7 \mathrm{cGy} \cdot \mathrm{h}^{-1}$ also was considered in the calculations. In addition to the dose calculation for prostate, the average received dose for bladder and rectum was also calculated and two cases of with and without GNP-loaded prostate were considered.

A humanoid computational phantom named as Korean man or (KTMAN-2) was utilized to simulate the seed implementation inside the prostate gland and organs at risks (Figure 3). This phantom contains 29 organs and 19 skeletal regions. It was produced from cross-sectional x-ray computed tomography images. It is made of $300 \times 150 \times 344$ voxels with a spatial resolution of $2 \times 2 \times 5 \mathrm{~cm}^{3}$. The anatomic properties of an average Korean man with a height of $171 \mathrm{~cm}$ and a weight of $70 \mathrm{~kg}$ have been considered in the structure of this computational phantom. For dose enhancement calculations, the voxels of the prostate gland were filled with GNPs using Lattice card in MCNPX code. The dose calculations were performed for voxels of prostate gland including two cases of with and without GNPs. 


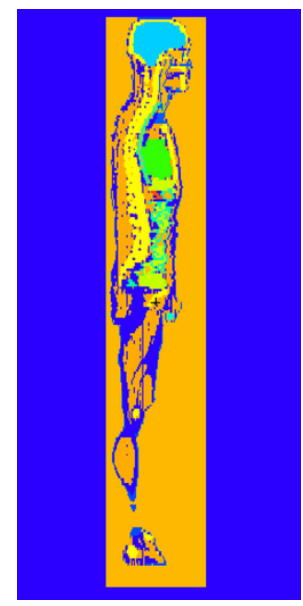

Figure 3a. The lateral view of Korean man computational phantom plotted using MCNPX code and plot from Korean man voxel phantom (KTMAN-2). The densities of bladder, prostate, and rectal wall were considered $1.023,1.025$, and $1.013 \mathrm{~g} / \mathrm{cm}^{3}$ respectively.

\section{Results and Discussion}

Dose rate constant, geometry and radial dose functions were calculated for the simulated seed. It was tabulated in Table $\mathbf{1 .}$ Comparing our study results with the other published results confirmed the accuracy of the simulated model for dose calculations. In the absence of GNPs, the prostate as the target organ absorbed $28.34 \mathrm{mSv} \cdot \mathrm{Ci}^{-1}$. Rectum, bladder, and urethra received dose were as small as $0.03 \mathrm{mSv} \cdot \mathrm{Ci}^{-1}, 0.02 \mathrm{mSv} \cdot \mathrm{Ci}^{-1}$, and $0.01 \mathrm{mSv} \cdot \mathrm{Ci}^{-1}$ respectively. According to the dose calculations, the ratios of target organ dose to the bladder and rectum were around $9.44 \times 10^{2}$ and $4.72 \times 10^{2}$. The obtained doses and ratios show that application of ${ }^{103} \mathrm{Pd}$ seeds for prostate brachytherapy is a suitable and safe method concerning the peripheral organs. Using GNPs in a concentration of $7 \mathrm{mg} \mathrm{Au} / \mathrm{g}$ changed the calculated doses considerably and the prostate dose enhanced up to $23 \%$. DEF was also calculated for a distance of 1 to $30 \mu \mathrm{m}$ around a single GNP. The results are shown in Figure 4. Calculated DEF in the first $\mu \mathrm{m}$ in the vicinity of a single GNP was $48 \%$ and dropped to $5 \%$ in $30 \mu \mathrm{m}$ from the GNP. The average DEF in the presence of all GNPs was approximately 23\%. This phenomenon showed a high dose gradient in the vicinity of the GNPs which can kill the cancer cell effectively in the site. In Table 1, the calculated dosimetric parameters of the simulated source were described. In the case of dose rate constant, the difference between our results and TLD measurement for the same model source was $24 \%$. This higher difference with measurement can be attributed to the measurement conditions such as the medium in which the results were obtained. Our calculation was performed in a humanoid phantom and the densities of bladder, prostate, and rectal wall were considered $1.023,1.025$, and $1.013 \mathrm{~g} / \mathrm{cm}^{3}$ respectively, while in the measurement study the perspex phantom with a density of 1.08 $\mathrm{g} / \mathrm{cm}^{3}$ was used.

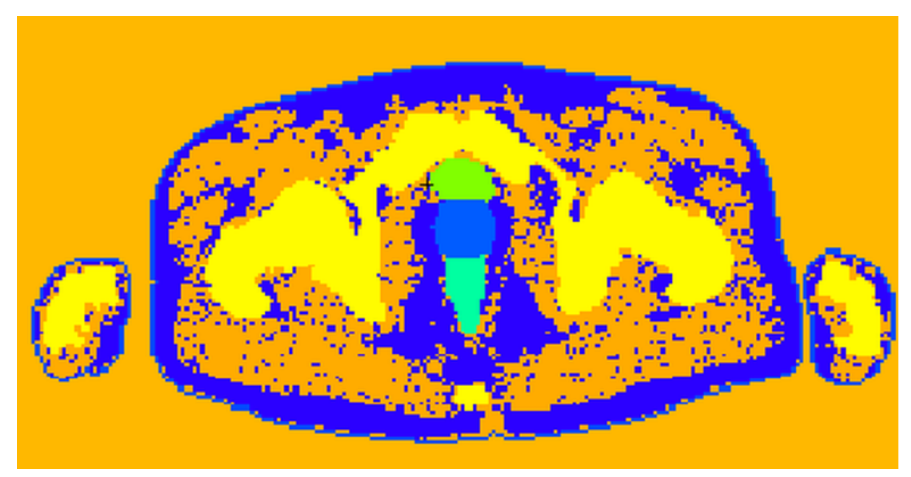

Figure 3b. The cross-sectional view of Korean man computational phantom plotted using MCNPX code and plot from Korean man voxel phantom (KTMAN-2). The densities of bladder, prostate, and rectal wall were considered 1.023, 1.025, and $1.013 \mathrm{~g} / \mathrm{cm}^{3}$ respectively. B: bladder and rectum were shown as organs at risk and P: prostate is source loaded target organ.

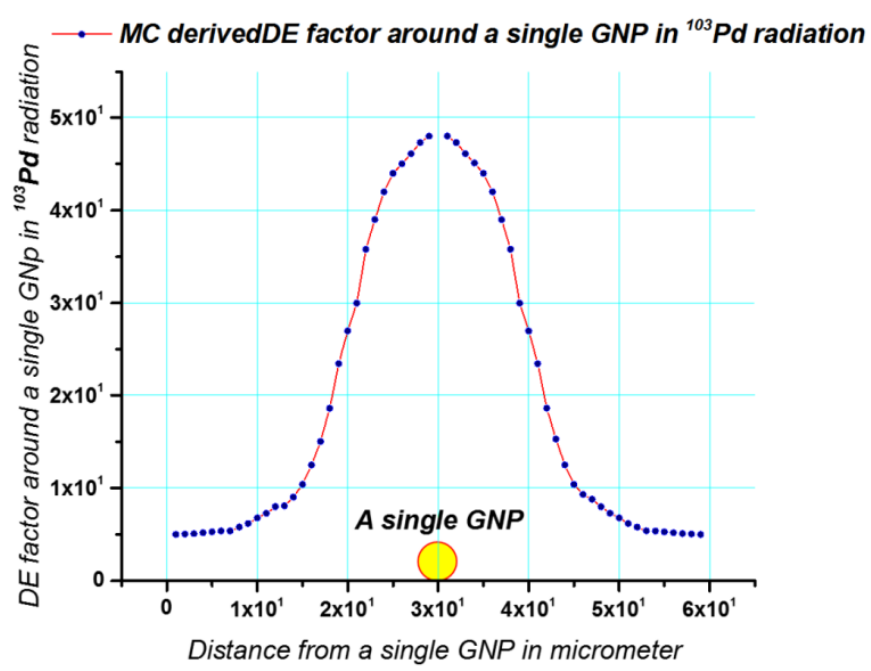

Figure 4. MC-derived dose enhancement factor around a single GNP.

Table 1. Dose rate constant $\Lambda\left(\mathrm{cGy}^{\circ} \mathbf{h}^{-1} \cdot \mathrm{U}^{-1}\right)$ calculated by MC simulation in this study and comparison with the experimental works. Our MC simulation result difference with TLD measurement: $24 \%$. Our MC simulation result difference with MC simulation: $3 \%$.

\begin{tabular}{lc}
\hline \hline \multicolumn{1}{c}{ Study } & $\begin{array}{c}\text { Dose rate constant } \mathbf{\Lambda} \\
\mathbf{c G y} \cdot \mathbf{h}^{\mathbf{1}} \cdot \mathbf{U}^{\mathbf{1}}\end{array}$ \\
\hline Our MC calculation in the humanoid phantom & $0.666 \pm 0.01$ \\
TLD dosimetry in Perspex [34] & $0.83 \pm 0.05$ \\
MC simulation in Liquid water [34] & $0.69 \pm 0.05$ \\
\hline \hline
\end{tabular}


Geometric parameters were calculated different radii including $\mathrm{r}=0.25 \mathrm{~cm}, 0.5 \mathrm{~cm}$, and $5 \mathrm{~cm}$. our results were in good agreement with the reported measured values of Raisali et al. [31]. Our simulations were performed at $15^{\circ}, 30^{\circ}, 45^{\circ}, 60^{\circ}$, and $90^{\circ}$ angles. The results were tabulated in Table 2 . It can be seen that a very small difference exists between our reported normalized geometry function and the other similar work [22,25,28,30,31]. Additionally, radial dose function was tabulated in Table 3 which includes TLD measured and MCderived values for the same source of ${ }^{103} \mathrm{Pd}$. Furthermore, the anisotropy factor for the simulated brachytherapy seed was obtained and compared with reported values in Table 4. Our results were compared with the MC study of Raisali et al. the [31]. There was a close agreement between our results and their reported values.

In the case of DEF of GNPs in the brachytherapy, some paper has been published [35,37-42]. The studies show that for low energy sources, DEF of nanoparticles is higher than high energy sources. It may be attributed to this fact that the photoelectric phenomenon is dominant in low energies and also photoelectrons produced from low energy photons deposit their energy in short range from the production site. Studying the dose distribution around a single GNP showed a dramatic DEF in a few $\mu \mathrm{m}$ distance from the GNP which creates a dose inhomogeneity inside target region as well as higher cell killing effect in the vicinity of GNPs.

\section{Conclusion}

We concluded that using the GNPs and low energy-high dose rate ${ }^{103} \mathrm{Pd}$ leads to significant $\mathrm{DEF}$ in brachytherapy of prostate cancer. Furthermore, a maximum dose enhancement of $48 \%$ plus dramatic dose gradient in micro-scale were found in the vicinity of GNPs which can cause higher cells kill effect in tumoral tissue compared to the conventional treatment without using GNPs. Our study showed ${ }^{103} \mathrm{Pd}$ with GNPs can be considered as an applicable choice for prostate brachytherapy.
Table 2. Normalized geometry function for the simulated seed in present work calculated by MC code and comparison with other work on the same model.

\begin{tabular}{lrrrrrr}
\hline \hline Distance $(\mathbf{R})=\mathbf{0 . 2 5} \mathbf{~ c m}$ & $\boldsymbol{\alpha}=\mathbf{0}^{\circ}$ & $\boldsymbol{\alpha}=\mathbf{1 5}^{\circ}$ & $\boldsymbol{\alpha}=\mathbf{3 0}^{\circ}$ & $\boldsymbol{\alpha}=\mathbf{4 5}^{\circ}$ & $\boldsymbol{\alpha}=\mathbf{6 0}^{\circ}$ & $\boldsymbol{\alpha}=\mathbf{9 0}$ \\
\hline Present work & 3.235 & 2.563 & 1.688 & 1.197 & 0.923 & 0.791 \\
Reference [27] & 3.245 & 2.574 & 1.691 & 1.202 & 0.925 & 0.793 \\
\hline \hline
\end{tabular}

Table 3. Radial dose function for the simulated IR01- ${ }^{103}$ Pd seed and comparison with the TLD measurement for the same model.

\begin{tabular}{lccc}
\hline \hline \multirow{2}{*}{ Study } & \multicolumn{3}{c}{$\mathbf{R}(\mathbf{c m})$} \\
\cline { 2 - 4 } & $\mathbf{0 . 5}$ & $\mathbf{1}$ & $\mathbf{5}$ \\
\hline $\begin{array}{l}\text { Our study; } \\
\text { In phantom }\end{array}$ & 1.013 & 1.001 & 0.19 \\
$\begin{array}{l}\text { Line geometry function; } \\
\text { Measurement medium was Perspex [27] }\end{array}$ & 1.07 & 1.00 & 0.19 \\
$\begin{array}{l}\text { Line geometry function; } \\
\text { MC derived in Perspex [27] }\end{array}$ & 1.012 & 1.00 & 0.19 \\
$\begin{array}{l}\text { Point geometry function derived by MC } \\
\text { simulation in liquid water_[27] }\end{array}$ & 1.173 & 1.00 & 0.092 \\
\hline \hline
\end{tabular}

Table 4. Anisotropy factor calculated in the present study in comparison with the measured and simulated values for IR01${ }^{103}$ Pd seed.

\begin{tabular}{|c|c|c|c|c|c|c|c|c|c|c|}
\hline & \multicolumn{10}{|c|}{ Methods } \\
\hline & \multicolumn{7}{|c|}{ The current study } & \multicolumn{3}{|c|}{$\begin{array}{c}\text { Measured values } \\
\text { in Perspex [27] }\end{array}$} \\
\hline & $\mathbf{0}^{\circ}$ & $15^{\circ}$ & $30^{\circ}$ & $45^{\circ}$ & $60^{\circ}$ & $75^{\circ}$ & $90^{\circ}$ & $\mathbf{0}^{\circ}$ & $30^{\circ}$ & $60^{\circ}$ \\
\hline $1 \mathrm{cn}$ & 0.5956 & 0.528 & 0.550 & 0.585 & 0.790 & 0.949 & 1.000 & 0.650 & 0.600 & 0.900 \\
\hline $2 \mathrm{~cm}$ & 0.685 & 0.592 & 0.674 & 0.801 & 0.904 & 0.968 & 1.000 & 700 & 720 & 950 \\
\hline $3 \mathrm{~cm}$ & 0.665 & 0.602 & 0.688 & 0.809 & 0.908 & 0.974 & 1.000 & 0.670 & 0.720 & 0.900 \\
\hline
\end{tabular}

\section{References}

[1] Yang R, Wang J, Zhang H. Dosimetric Comparison of Permanent Prostate Brachytherapy Plans Utilizing Cs-131, I-125 and Pd-103 Seeds. Cancer Biother Radiopharm. 2009;24(6):701-5.

[2] Ververs J, Anscher M, Rivard M, Todor D. A Treatment Planning Feasibility Study for Prostate LDR Brachytherapy Treatments Using the New 103-Pd CivaString Source. Comparison with Clinical Cases Using the TheraSeed Model 200 103-Pd Source. Med Phys. 2013;40(6):310.

[3] Rivard MJ, Reed JL, DeWerd LA. 103Pd strings: Monte Carlo assessment of a new approach to brachytherapy source design. Med Phys 2014;41(1):011716.

[4] Chandran PR, Thomas RT. Chapter 14 - Gold Nanoparticles in Cancer Drug Delivery. In: Ninan STG, editor. Nanotechnology Applications for Tissue Engineering.Oxford: William Andrew Publishing; 2015; 221-37.

[5] Gilles M, Brun E, Sicard-Roselli C. Gold nanoparticles functionalization notably decreases radiosensitization through hydroxyl radical production under ionizing radiation. Colloids Surf B: Biointerfaces 2014;123:770-7.

[6] Xie WZ, Friedland WF, Li WB, et al. Simulation on the molecular radiosensitization effect of gold nanoparticles in cells irradiated by x-rays. Phys Med Biol. 2015;60(16):6195-212. 
[7] Yao XF, Huang CF, Chen XF, et al. Chemical Radiosensitivity of DNA Induced by Gold Nanoparticles. J Biomed Nanotechnol. 2015;11(3):478-85.

[8] Alexis F, Rhee JW, Richie JP, et al. New frontiers in nanotechnology for cancer treatment. Urologic Oncology: Seminars and Original Investigations. 2008;26(1):74-85.

[9] Gao Z, Zhang L, Sun Y. Nanotechnology applied to overcome tumor drug resistance. J Control Release 2012;162(1):45-55.

[10] Geso M. Nanoparticle augmented radiation treatment decreases cancer cell proliferation. Nanomedicine: Nanotechnology, Biology and Medicine 2013;9(2):302-3.

[11] Joh DY, Kao GD, Murty S, et al. Theranostic Gold Nanoparticles Modified for Durable Systemic Circulation Effectively and Safely Enhance the Radiation Therapy of Human Sarcoma Cells and Tumors. Transl Oncol. 2013;6(6):722-732.

[12] Nazir S, Hussain T, Ayub A, et al. Nanomaterials in combating cancer: Therapeutic applications and developments. Nanomedicine: Nanotechnology, Biology and Medicine. 2014;10(1):19-34.

[13] Bertrand N, Wu J, Xu X, et al. Cancer nanotechnology: The impact of passive and active targeting in the era of modern cancer biology. Adv Drug Deliv Revi. 2014;6666:2-25.

[14] Brede C, Labhasetwar V. Applications of Nanoparticles in the Detection and Treatment of Kidney Diseases. Adv Chronic Kidney Dis. 2013;20(6):454-65.

[15] Chatterjee DK, Fong LS, Zhang Y. Nanoparticles in photodynamic therapy: An emerging paradigm. Adv Drug Deliv Rev 2008;60(15):1627-37.

[16] Etame AB, Diaz RJ, O'Reilly MA, et al. Enhanced delivery of gold nanoparticles with therapeutic potential into the brain using MRIguided focused ultrasound. Nanomedicine: Nanotechnology, Biology and Medicine. 2012;8(7):1133-42.

[17] Feng G, Kong B, Xing J, Chen J. Enhancing multimodality functional and molecular imaging using glucose-coated gold nanoparticles. Clin Radiol. 2014;69(11):1105-11.

[18] Gaca S, Reichert S, Multhoff G, et al. Targeting by cmHsp70.1-antibody coated and survivin miRNA plasmid loaded nanoparticles to radiosensitize glioblastoma cells. J Control Rel. 2013;28;172(1):201-6.

[19] Hong H, Chen F, Zhang Y, Cai W. New radiotracers for imaging of vascular targets in angiogenesis-related diseases. Adv Drug Deliv Rev. 2014;76:2-20.

[20] Lin Y, McMahon SJ, Scarpelli M, et al. Comparing gold nano-particle enhanced radiotherapy with protons, megavoltage photons and kilovoltage photons: a Monte Carlo simulation. Phys Med Biol. 2014;59(24):7675-89.

[21] Asadi S, Vaez-Zadeh M, Vahidian M, et al. Ocular brachytherapy dosimetry for 103Pd and 125I in the presence of gold nanoparticles: a Monte Carlo study. J Appl Clin Med Phys. 2016;17(3):90-99.

[22] Khosravi H, Hashemi B, Mahdavi SR, Hejazi P. Effect of Gold Nanoparticles on Prostate Dose Distribution under Ir-192 Internal and 18 MV External Radiotherapy Procedures Using Gel Dosimetry and Monte Carlo Method. J Biomed Phys Eng. 2015;5(1):3-14.

[23] Sinha N, Cifter G, Sajo E, et al. Brachytherapy application with in situ dose painting administered by gold nanoparticle eluters. Int J Radiat Oncol Biol Phys. 2015;91(2):385-92.

[24] Reed JL, Rivard MJ, Micka JA, et al. Experimental and Monte Carlo dosimetric characterization of a 1 cm 103Pd brachytherapy source. Brachytherapy. 2014;13(6):657-67.

[25] P Saidi, M Sadeghi, M Enferadi, G Aslani. Investigation of palladium-103 production and IR07-103Pd brachytherapy seed preparation. Ann Nucl Energy. 2011;38(1):2168-73.

[26] Butler WM, Merrick GS. Focal prostate brachytherapy with 103Pd seeds. Phys Med. 2016;32(3):459-64.

[27] Li ZY, Gao HB, Deng XS, et al. Preparation of 103Pd brachytherapy seeds by electroless plating of 103Pd onto carbon bars. Appl Radiat Isot. 2015;103:128-30.

[28] Saidi P, Sadeghi M, Shirazi A, Tenreiro C. Dosimetric parameters of the new design 103Pd brachytherapy source based on Monte Carlo study. Phys Med. 2012;28(1):13-8.

[29] National Laboratory Report No. BNL.NCS-17541. Cross section Evaluation Working Group. ENDF/B-VI summary documentation (ENDF-201). December 2000: National Nuclear Data Center; 2000.

[30] Rivard MJ, Coursey BM, DeWerd LA, et al. Update of AAPM task group No. 43 report: a revised AAPM protocol for brachytherapy dose calculations. Med Phys. 2004;31(3):633-74.

[31] Raisali G, Ghonchehnazi MG, Shokrani P, Sadeghi M. Monte Carlo and experimental characterization of the first AMIRS 103Pd brachytherapy source. Appl Radiat Isot 2008;66(12):1856-60.

[32] Kim YJ, Park JH, Yun IH, Kim YS. A prospective comparison of acute intestinal toxicity following whole pelvic versus small field intensity-modulated radiotherapy for prostate cancer. Onco Targets Ther. 2016;9:1319-25.

[33] Xie WZ, Friedland WF, Li WB, et al. Simulation on the molecular radiosensitization effect of gold nanoparticles in cells irradiated by x-rays. Phys Med Biol 2015; 21;60(16):6195-212. 
[34] Yang CJ, Chithrani DB. Nuclear Targeting of Gold Nanoparticles for Improved Therapeutics. Curr Top Med Chem. 2016;16(3):27180.

[35] Brun E, Sanche L, Sicard-Roselli C. Parameters governing gold nanoparticle X-ray radiosensitization of DNA in solution. Colloids Surf B Biointerfaces. 2009;7(1)2:128-34.

[36] Brun E, Duchambon P, Blouquit Y, et al. Gold nanoparticles enhance the X-ray-induced degradation of human centrin 2 protein. Radiat Phys Chem. 2009;78(3):177-83.

[37] Feng G, Kong B, Xing J, Chen J. Enhancing multimodality functional and molecular imaging using glucose-coated gold nanoparticles. Clin Radiol. 2014;69(11):1105-11.

[38] Ghorbani M, Mehrpouyan M, Davenport D, Ahmadi Moghaddas T. Effect of photon energy spectrum on dosimetric parameters of brachytherapy sources. Radiol Oncol. 2016;50(2):238-46.

[39] Reynoso FJ, Manohar N, Krishnan S, Cho SH. Design of an Yb-169 source optimized for gold nanoparticle-aided radiation therapy. Med Phys. 2014;41(10):101709.

[40] Sinha N, Cifter G, Sajo E, et al. Brachytherapy application with in situ dose painting administered by gold nanoparticle eluters. Int J Radiat Oncol Biol Phys. 2015;91(2):385-92. 